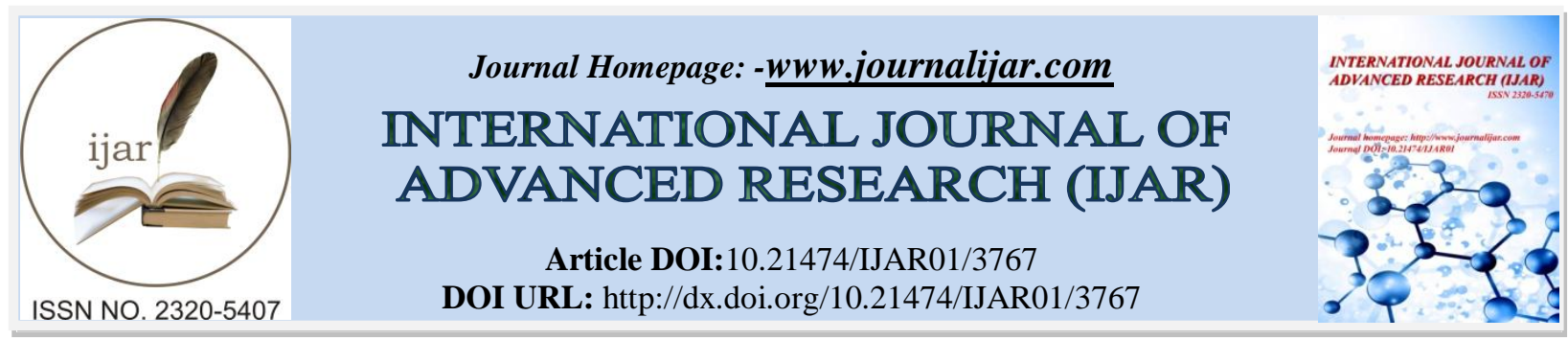

RESEARCH ARTICLE

\title{
GASTROPROTECTIVE EFFECT OF A NEW FORMULATED MILK TABLET ON ETHANOL- INDUCED GASTRIC MUCOSAL INJURY IN RATS.
}

\author{
${ }^{*}$ Enas A. El-Hussieny ${ }^{1}$, EmanF.Mohamed ${ }^{2}$, NeamahR.Attala ${ }^{2}$ and Fawzia A. A.Abd El-Rahman ${ }^{1}$. \\ 1. Zoology Department, Faculty of Science, Ain Shams University, Cairo, Egypt. \\ 2. Dairy Technology Department, Animal Production Research Institute, Agricultural Research Center, Giza, \\ Egypt.
}

\section{Manuscript Info}

Manuscript History

Received: 17 January 2017

Final Accepted: 03 February 2017

Key words:-

NewFormulated Milk Tablet (FM), Gastroprotective, Ethanol, Antioxidant, Anti-inflammatory, Lipid peroxidation. (n..........................

Published: March 2017

\begin{abstract}
FM is a newly formulated milk tablet that provides the supplementary food such as milk and honey, also fortified it with high nutritive value components (bee pollen, palm pollen, Royal jelly, Propolis, Ginseng,and Bee pollen) that have many remarkable biological activities such as antioxidant, antibacterial and anti-inflammatory features.The current study aimed to investigate the potential gastroprotective effects of FM against ethanol-induced gastric injury in male albino rats. The experimental set included four groups; Control group, an ulcer control group treated with absolute ethanol $(10 \mathrm{ml} / \mathrm{kg})$, FM pretreated groups at doses (157 and $314 \mathrm{mg} / \mathrm{kg}$ per oral). The gastric secretion was collected for assessment of $\mathrm{pH}$. The involvement of malondialdehyde (MDA), catalase (CAT), nitric oxide (NO) and tumor necrosis factor-alpha (TNF- $\alpha$ ), alanine aminotransferase(ALT), aspartate aminotransferase (AST), alkaline phosphatase (ALP), Uric acid, and creatinine were also measured. Histopathological studies were undertaken to evaluate the influence of the treatment on the gastric wall mucosal layer. FM suppressed gastric inflammation induced as a result of ethanol administration by decreasing of tumor necrosis factor- $\alpha$ (TNF- $\alpha$ ), nitric oxide (NO) and neutrophil infiltration. Meanwhile, FM halted gastric oxidative stress via reduction of MDA level with concomitant enhancement of the activity of a potent antioxidant CAT. These favorable actions were associated with attenuation of the severity of ethanol gastric mucosal injury as evidenced by the morphological and histological observations. Together, these findings postulated the potent gastroprotective action of FMagainst ethanol-induced gastric injurywhichwas mediated via suppression of gastric inflammation and oxidative stress besides boosting of the antioxidant defense.
\end{abstract}

Copy Right, IJAR, 2017,. All rights reserved.

\section{Introduction:-}

Gastrointestinal ulcer disease poses a serious economic load as a treatment for the ulcers account to at least $10 \%$ of the total cost of treating digestive disorders (Brown and Everhart, 1994; Sandler et al., 2002). Peptic ulcer is a common disorder of the entire gastrointestinal tract. Moreover, about 8 to $10 \%$ of the global population affected by peptic ulcers, approximately 5\% of them suffer from gastric ulcers (Calam and Baron, 2001). The ulcers that affect 
the gastrointestinal system are usually aggravated by a disproportion between destructive and defensive factors in the stomach (Calam and Baron, 2001). The major causes of peptic ulcer disease are stress, alcohol, chronic use of non-steroidal anti-inflammatory drugs $\left(\mathrm{NSAID}_{\mathrm{S}}\right)$, cigarette smoking, genetic predisposition, diet, and Helicobacter pylori infection(Nemeroff ,1982).

Alcohol consumption has been commonly associated with gastric mucosal lesions including gastritis, gastric ulcer, and even gastric carcinoma (Franke et al., 2005). The mechanisms underlying ethanol-induced gastric ulcer have not been fully defined. Yet, mounting evidence has indicated that oxidative stress, proinflammatory cytokines and apoptosis play crucial roles in its pathogenesis (Al Batranet al., 2013; Mei et al., 2012; Sangiovanniet al., 2013). Ethanol is widely used to induce experimental gastric ulcer in animals(Loguercioet al.,1993).The experimental model of ethanol-induced gastric injury mimics several features of the human condition and thus provides a mean for assessing agents with potential anti-ulcer actions along with their implicated mechanisms for gastric protection (Liu et al., 2012; Salgaet al.,2012). The administration of these drugs has been associated with several adverse effects such as nausea, constipation, gynecomastia and impotence that limit their use (Halabiet al., 2014). Besides, most of these drugs, lackan effect on other factors involved in ulcer disease and therefore, do not achieve all treatment goals. Thus, the search for effective agents with fewer side effects has been regarded as an effective strategy for the management of gastric ulcers (Antonisamyet al., 2014; Halabiet al., 2014). Previously, agents with antioxidant features have displayed beneficial actions in protecting against alcohol-induced gastric ulcer (Antonisamyet al., 2014; Liu et al., 2012; Mei et al., 2012). Hence, there is a strong need for more effective antioxidant, and safe anti-ulcer agents with different protective mechanisms from natural resources.

The primary function of milk is to meet the complete nutritional requirements of human, making it a nutrient dense food. The recognition of milk's richness in nutrients that play a key-role in human physiology has in recent years led to a wide variety of novel applications in dairy technology (Livney,2010).

New formula of healthier milk tablet aims to improve nutritional status and health.An optimum formula of milk tablet was formulated to minimize cost and maximize nutritional value. Providing the supplementary food such as milk and honey and fortified it with a high nutritive values component (Skim milk powder, honey, bee pollen, palm pollen, Royal jelly, propolis and ginseng). The advantages of new formulated milktablets with high nutritive value are long shelf-life, convenience, and suitable for the distribution to remote areas. Skimmed milk powder is widely used due to its easy storage facility and immediate availability on hand in all conditions. Milk is a very important substitute for food in most circumstances. So using of skimmed milk powder is safer in such conditions as it contains all the nutritional constituents of calcium, proteins, many vitamins and carbohydrates and no fat.

Most people think of honey as excellent food, but some others consider it as medicine (Zaghloulet al., 2001), but only recently its antioxidant property has come to limelight. With increasing demand for antioxidant supply in the food, honey is becoming important source of antioxidant since it is rich in flavonoids and phenolic acids and other antioxidants including glucose oxidase,catalase, ascorbic acid, carotenoid derivatives, and organic acids. The antioxidants have several preventative effects against different diseases like cancer, cardiovascular diseases, inflammatory disorders, neurological degeneration, wound healing, infectious diseases and aging, which led to search for foods rich in antioxidants (Khalil etal.,2010). Beside all previous benefits for honey it could be as a natural binder for dairy product ingredients.

Bee pollen consider as a valuable therapeuticproduct greatly appreciated by the natural medicine because of its potential medical and nutritional applications. It demonstrates a series of actions such as anti-inflammatory, hepatoprotective, and immune-stimulating. The use of bee pollen as food supplement has been proposed to ameliorate various inflammatory diseases (Martín-Muñoz et al., 2010). However, there are very few studies about using bee pollen in food and dairy industry. On the other side, palm pollen grains are a good economic nutritional source can be used as human food supplements (Fernández,1983).

Royal jelly (RJ) is a secretion of the cephalic glands of nurse bees and serves as the most important part of honeybee larvae diet, playing a major role in caste differentiation (Moritz and Southwick, 1992). RJ, one of the most effectual and beneficial remedy for human beings, is widely used both in folk and in official medicine and it is a controversial dietary supplement. Due to its complex composition (water, proteins, lipids, carbohydrates, amino acids, mineral salts, vitamins, enzymes, hormones, and natural antibiotics), RJ has a multitude of pharmacological activities: antioxidant, hypoglycemia, hepatoprotective, antitumor, antibiotic,and anti-allergic (Mărghitaş, 2008). Moreover, 
propolisacts as an antibacterial factor. In addition, propolis is a powerful antioxidant (Özcan and Ayar, 2003). These effects are due to its high concentration of phenolics and other antioxidant compounds. Ginseng, the root of Panax species, is a well-known folk medicine. It has been used as traditional herbal medicine in China, Korea and Japan for thousands of years and today it is a popular and worldwide used natural medicine. The active ingredients of ginseng are ginsenosides, which are also called ginseng saponins (Kamal et al., 2016). Research suggests that the bioactive components of ginseng, such as ginsenosides and polysaccharides, can decrease oxidative stress, which is a mechanism associated with reduced chronic disease development (Sun, 2007; Uno and Nicholls,2010).

Yet, theimpact of thisnewly formulated milk tablet (FM) on gastric ulcer has not been previously explored. Thus, the present study aimed to investigate the potential gastroprotective actions of FM in ethanol-induced gastric injury in rats.

\section{Materials and Methods:- \\ Chemicals}

All common chemicals used were purchased from one of the following suppliers Sigma Co. (St. Louis, MO, USA). All other reagents were of the highest grade commercially available. (All chemicals used in the study were of analytical grade).

\section{Experimental Animals}

Adult male rats, weighing $(150 \pm 20) \mathrm{g}$. They were kept under observation for about 15 days before the onset of the experiment to exclude any infection. The chosen animals were housed in plastic cages with good aerated covers at $25^{\circ} \mathrm{C} \pm 0.5^{\circ} \mathrm{C}$ as well as $12 \mathrm{~h} \mathrm{light/dark} \mathrm{cycles.} \mathrm{Animals} \mathrm{were} \mathrm{allowed} \mathrm{free} \mathrm{access} \mathrm{to} \mathrm{water} \mathrm{and} \mathrm{were} \mathrm{supplied} \mathrm{daily}$ with a standard diet. The study design was approved by Ain Shams University Research Ethics Committee.

\section{Preparation of new formulated milk tablet (FM)}

The ingredients of FM consist of skim milk powder with different proportion as 50,55,60,65and 70\% in parallel with honey proportion as $28,23,18,13$ and $8 \%$, respectively then added bee pollen $10 \%$, palm pollen $5 \%$, Royal jelly $5 \%$, propolis $1 \%$ and ginseng $1 \%$ for each formula. Preparation of FM was prepared in the Dairy Technology Department Animal Production Research Institute. All ingredients were mixed then compressed into tablet form. Milk tablet samples were packed into airtight plastic bags and stored in the refrigerator for further analysis (AOAC, 2006). The chemical composition of milk tablet ingredients and Formulations of milk tablet from mixture design was shown in tables $(1,2)$.In addition, chemical composition of selected formula of new formulate milk tablet was illustrated in Table (4).

\section{Mucosal injury induction}

Gastric ulceration was induced in the animals according to the procedure described by (Huang et al., 2014). Briefly, rats were deprived of food but had free access to water $24 \mathrm{~h}$ prior to ulcer induction then, they were administered, with a single oral dose of $75 \%$ ethanol (10 $\mathrm{ml} / \mathrm{kg}$ body weight). Two hours after ethanol administration, animals were sacrificed.

\section{Animal grouping and treatments}

Thirty two male rats were randomly divided into four groups ( $\mathrm{n}=8$ per group): Group 1 (normal control) animals received only distilled water. Rats in group 2(ulcerated control) were given only $75 \%$ ethanol $(10 \mathrm{ml} / \mathrm{kg})$. Group 3 and 4 animals were given FM at doses $157 \mathrm{mg} / \mathrm{kg}$ and $314 \mathrm{mg} / \mathrm{kg}$, respectively, for 4 weeks, thenat day $29,75 \%$ ethanol $(10 \mathrm{ml} / \mathrm{kg})$ was given orally to each animal to induce gastric ulceration. Two hours after ethanol administration, all rats were sacrificed and stomachs were removed, opened along the greater curvature and gastric content was drained into a centrifuge tube.

\section{Blood Samples preparation}

Blood was collected from sacrificed rat in vacutainer and centrifuged $3000 \mathrm{rpm}$ for $10 \mathrm{~min}$. Plasma samples were collected and stored at -80 until used for biochemical analysis.

\section{Collection of gastric juice for determination of gastric $\mathrm{pH}$}

Gastric juice from each animal was centrifuged at $1000 \mathrm{rpm}$ for 10 minutes after adding $5 \mathrm{ml}$ distilled water to remove any solid debris and the volume of the supernatant was measured. The supernatant was then assayed for determination of the $\mathrm{pH}$ of gastric juice using a pH meter (Sabiu et al.,2015). 


\section{Preparation of stomach homogenate for biochemical investigations}

The gastric tissue was homogenized in $10 \mathrm{ml}$ ice-cold phosphate buffer (PBS, $100 \mathrm{mM}, \mathrm{pH}$ 7.4). The homogenized tissue (1g of tissue $/ 10 \mathrm{ml} \mathrm{PBS}$ ) was centrifuged at 4000rpm for 15 minutes. The supernatant was removed and stored at $-80^{\circ} \mathrm{C}$ for determination of oxidative stress markers and cellular defenses markers.

\section{Assessment of oxidant/antioxidant activities}

The determination of gastric mucous membrane lipid peroxides, expressed as malondialdehyde (MDA), was carried out according to the thiobarbituric acid assay (Liu et al.,2012). Using the TBA commercial kit (spectrum diagnostics Egyptian company of biotechnology, Cairo, Egypt). Catalase (CAT), and nitric oxide (NO) levels were tested in the gastric tissue homogenate using the CAT and the NO assay kits (spectrum diagnostics Egyptian company of biotechnology, Cairo, Egypt), following the manufacturer's instructions.

\section{Assessment of TNF- $\alpha$ level}

The plasma TNF- $\alpha$ level was measured using the Rat TNF-alpha (TNF- $\alpha$ ) ELISA kit (KOMA BIOTECH INC., Seoul, Korea) according to the manufacturer'sinstructions.

\section{Assessment of the liver and kidney functions}

The functioning of the liver was assayed by evaluating the alanine aminotransferase, aspartate aminotransferase, alkaline phosphatase, markers of hepatocellular damage, were established by colorimetric assay using a commercial kit (spectrum diagnostics Egyptian company of biotechnology, Egypt). The functioning of the kidney was assayed by evaluating the creatinine and uric acid by colorimetric assay using a commercial kit (spectrum diagnostics Egyptian company of biotechnology, Egypt).

\section{Histological evaluation of gastric injury}

From all experimental groups, the stomach of each rat was removed, opened along its greater curvature and rinsed with saline to remove the gastric contents. The stomach was stretched on a piece of cork with the mucosal surface facing upward and was then photographed with a digital camera.The histological evaluation of the gastric injury was performed on the specimens of the glandular stomach of three rats randomly selected from each group of the experiment. In brief, stomachs were fixed in the aqueous Bouin's fluid, embedded in paraplast, and sections were made at a thickness of 5-7 $\mu \mathrm{m}$ and stained with hematoxylin and eosin (Humason, 1972).

\section{Statistical analysis}

Statistical evaluation was conducted with Instat Program GraphPad. Software, Inc, San Digeo, USA, version3. 6, Copyright@1992-2003 Results were expressed as mean \pm SEM. The results were analyzed for statistical significance by one way ANOVA followed by Tukey-Kramer multiple comparison post-test. Values of $p<0.05$ were regarded as significant.

\section{Results:-}

Sensory acceptability Consumer acceptance test for products form and mixture design experiment:Consumer acceptance test was performed at the Animal Production Research Institute as presented in Table (3) it could be observed that the rate between the milk powder and honey had a highly effect in all sensory attributes. Formulations (E) and (A) showed the lowest scores in the overall acceptability, it wasrecorded 70 and 76 points. Highest percent of skim milk powder cooperates with the lowest percent of honey in the formula (E) affected tablets properties as hard tablets with weak and pail flavor. On the other hand the lowest percent of skim milk powder and the highest percent of honey as formula (A) gave weak form and body and over sweetness flavor. More acceptance scores recorded by formulas (B) and (D) respectively. From the same table it could be observed that formula (C) gained the highest scores owing to its balance in the all nutritive value and overall sensory acceptability.

\section{Effect of FM on PH:-}

Fig.1a shows the effect of (FM) on gastric secretions of ethanol ulcerated rats. EtOH administration caused significant $(\mathrm{p}<0.05)$ increase in $\mathrm{pH}$ value as compared to control group. Pre-treatment with the FM at both doses produced insignificant increase in $\mathrm{pH}$ value when compared with ulcerated control rats. While a significant increase was obsereved $(\mathrm{p}<0.001)$ in both doses as compared to control group. 


\section{Effect of (FM) on biochemical parameters of stomach tissue:-}

The gastroprotective effect of $157 \mathrm{mg} / \mathrm{kg}$ and $314 \mathrm{mg} / \mathrm{kg}$ doses of $(\mathrm{FM})$ on the ethanol-induced gastric ulcer was estimated with various gastric parameters. There were remarkable changes in the gastric parameters of ulcerated and (FM) pre-treated groups as compared with the control animals.

\section{Evaluation of Antioxidant and enzymatic activities:-}

The effect of (FM) on the ulceration process in ethanol-induced ulcer was evaluated by the oxidative stress marker level, MDA, catalase activity and gastric NO (Fig.1).

Administration of ethanol triggered an oxidative stress as indicated by significant increase of MDA level ( $\mathrm{p}<0.001)$ as compared to control group. Pretreatment with both concentrations of (FM) significantly protect the gastric tissue against the oxidative stress as evidenced by significant $(\mathrm{p}<0.05, \mathrm{p}<0.001$ respectively) lowering of MDA as compared to ulcer control rats. On the other hand, the gastric ulcer induced group showed a significant increase $(\mathrm{p}<0.001)$ in catalase activity in comparison to the normal control. Pretreatment with FM, showed a significant $(\mathrm{P}<$ $0.05)$ reductionin the catalase activity at the low dose, and a significant increaseat the high $\operatorname{dose}(\mathrm{P}<0.05)$ as compared to ulcer group. While, both doses of $(F M)$ significantly significant elevate $(p<0.001)$ the enzyme level as compared to control group. Rats subjected to EtOH administration (ulcer control rats) showed significantly decreased NO content $(\mathrm{p}<0.05)$ as compared to normal control rats. (FM) pretreatment at both doses significantly increased NO content ( $<<0.001, \mathrm{p}<0.001$ respectively) as compared to ulcer group.

Evaluation of plasma TNF- $\alpha$ level:-

Administration of ethanol resulted in a robust inflammatory response as indicated by a marked elevation of serum TNF- $\alpha(\mathrm{p}<0.001)$ as compared to the control group (Fig.1c). Pretreatment with (FM) in both doses significantly $(\mathrm{P}<0.001)$ lowered the levels of TNF- $\alpha$ when compared to ethanol ulcer group.

\section{Evaluation of liver function:-}

EtOH administration did not significantly affect the levels of AST, ALT, and ALP as compared to control group (Fig.2a). (FM) pretreatment at high dose significantly ( $p<0.05$, Fig.2a) decrease AST level as compared to control rats. In addition, both doses of (FM) showed a significant ( $p<0.01, p<0.05$, respectively) decrease of ALP level as compared to ulcer control rats. (Fig.2a).

\section{Evaluation of kidney function:-}

EtOH administration did not significantly affect the levels of creatinine, and uric acid as compared to control group (Fig.2b). (FM) pretreatment at low dose significantly ( $\mathrm{p}<0.05$, Fig $2 \mathrm{~b}$ ) increased the uric acid level as compared to ulcer control rats, while no significant change was observed in creatinine level at both doses of (FM) as compared to ulcer control rats (Fig.2b).

\section{Microscopic Evaluation of the gastric injury:-}

The morphological observations of the stomach of the control group showed that it is pink in colour without any hemorrhagic areas and its mucosa is thrown into prominent longitudinal folds, rugae, (Fig. 3a). Moreover, the ethanol induced gastric injury in the ulcer group that include hyperemia and multiple linear hemorrhagic red bands of different size along the glandular portion of the stomach indicating that the gastric ulcer was completely formed (Fig. 3b). In addition, the FM pre-treatment groups exhibited a dose-dependent protective effect against ethanolinduced gastric injury, with a marked decrease in the gastric mucosa injury and a significant ulcer inhibition compared with the ulcer group at the standard dose and maximal ulcer inhibition at the double standard dose (Figs. $3 \mathrm{c}$ and $3 \mathrm{~d})$.

The histological observations of the gastric mucosa of the control group revealed that it consists of the lining epithelium, lamina propria and muscularis mucosae (Fig. 4a). The lining epithelium is formed of simple columnar epithelial cells (Fig. 4b). In addition, the lamina propria consists of connective tissue that contains numerous straight tubular gastric glands (Fig. 4a). These glands are lined by three main types of cells; the luminal lining epithelium, oxyntic and peptic (chief or zymogenic) cells (Figs. 4b and 4c). Moreover, the muscularis mucosae is built of smooth muscle fibers (Fig. 4a).

The ethanol induced extensive gastric mucosal lesions in the ulcer group which extended to the two internal thirds or the entire mucosal thickness (Fig. 5a). These lesions included highly necrotic mucosa, epithelial lifting, mucosal 
hemorrhage and leukocyte infiltration in the lamina propria and submucosa (Figs. 5a-5c). Moreover, some mucosal cells showed necrosis (Fig. 5d) and others possess pyknotic nuclei (Fig. 5e).

The mucosal damage induced by absolute ethanol was significantly decreased by FM pre-treatment and the mucosa was relatively intact. FM has been shown to exert the cytoprotective effects in a dose-dependent manner. The rats pre-treated with standard diet of FM showed markedly better reductions in gastric lesion and comparatively better protection of the gastric mucosa, (Figs. 6a-6c), as proven by very superficial injury only, (Fig. 6d), and reduction of leukocyte infiltration (Fig. 6a). Moreover, rats treated with a double standard dose of FM showed more or less normal histological architecture of the gastric mucosa (Fig. 7a) and demonstrated significantly better mucosal protection and absence of leukocyte infiltration (Figs. 7b and 7c).

\section{Discussion:-}

Milk made formulations provide a simple, novel and patient friendly approach for the delivery of ionized as well unionized hydrophobic drugs. Incorporation of milk in the formulation results in solubilization of drugs with enhanced pharma- kinetic properties. Taste masking and gastroprotective properties also prove it a promising carrier for drugs. Further in-vitro studies are yet to be carried out to elucidate the colloidal stability of resulting emulsion, particle size and distribution of milk component (Kamal et al., 2016).

The current study highlights, for the first time, the protective role of FM, a new formulated milktablet, against ethanol-induced gastric injury in rats. The ethanol is commonly used for inducing ulcer in the experimental rats and leads to intense gastric mucosal injury. Furthermore, alcohol causes indirect destructive effects via the recruitment of leukocytes which drives oxidative stress, inflammatory responses and apoptosis (Arabet al., 2015).

In the present investigation, FM treatment markedly enhances the gastric mucosa and attenuates the gastric injury such as hemorrhage and hyperemia. These results suggest that FM has a protective action by reducing the gastric mucosal injury exposed to ethanol.

Additionally, secretion of mucus is supposed to be such as vital protective factor against gastric injuries. Previous reports suggested that mucus delays the diffusion of protons, which would aid in maintaining a favorable $\mathrm{pH}$ at the epithelial apical surface (Schreiber and Scheid, 1997).The pH gives an idea of the level of acidity and volume of gastric secretions. Low $\mathrm{pH}$ value is anindicator of decreased hydrogen ion concentration in gastric juice. This has been linked to the pathogenesis of ulcer and gastric damage in experimental animals (Lüllmannet al., 2000). The present study showed that FM neutralized stomach juice $\mathrm{pH}$ to $(7.30 \pm 0.33,7.24 \pm 0.77$; respectively) by significantly increases $(\mathrm{P}<0.001)$ of the $\mathrm{pH}$ value of the gastric contents at both doses.

The involvement of oxidative stress in the pathogenesis of ethanol-induced gastric injury has been confirmed by several studies. One of the possible mechanisms of EtOH induced acute gastric mucosal injury is thought to arise as a result of extreme generation of free radicals(Schlorffet al., 1999). The free radicals have a high tendency to react rapidly with lipids, thus, triggering lipid peroxidation that can lead to disorganization of the membrane and consequently reduces its fluidity(Recknagel and Ghoshal, 1996).

In harmony with these mentioned data, the current study showed that, ethanol administration initiated gastric oxidative stress and significantly increased the MDA level,a byproduct of lipid peroxidation, as compared to control group. While, this elevation of MDA level was reversed by pretreatment with FM in both doses ( $<<0.05, p<0.001$ respectively) suggesting a reduction in the lipid peroxidation activity upon the oral administration of FM.

Catalase, a highly reactive enzyme, plays a protective antioxidant effect against the harmful actions of lipid peroxidation by reacting with $\mathrm{H}_{2} \mathrm{O}_{2}$ to form water and molecular oxygen (Zakariaet al., 2015). Data of the current study demonstrated that FM combated oxidative stress and enhanced the antioxidant status in animals with ethanol gastritis as revealed by the reduction of MDA levels. This was accompanied with a significant $(\mathrm{P}<0.001)$ increase of CAT in both doses. These findings highlight the premise that the antioxidant features of the components of this new formulated milktablet are implicated in the alleviation of ethanol gastric injury. In fact, scavenging of ROS has been considered as one of the mechanisms involved in the healing of ulcers (Mei et al., 2012), which confirmed that FMantiulcerogenic properties are partially based on its antioxidant function.

Emerging evidences support the concept that the elevation of NO levels is a protective mechanism against the damage of gastric mucosa (J'unioret al.,2014; Nordinet al.,2014). It is considered one of the most important 
defensive endogenous mediators in the gastric mucosa and a potent chain-breaking antioxidant in free radicalmediated lipid Peroxidation(O’Donnell et al., 1997; Halabiet al., 2014). Additionally, NO plays avital role in maintaining the gastric mucosal alkaline production(Calatayudet al., 2001), and inhibiting neutrophil aggregation (Wallace et al., 1997).

According to our results, EtOH administration significantly $(\mathrm{P}<0.05)$ decreased NO content in the stomach. Similarly, as reported by (Abdulla et al., 2009; Goswamiet al., 2011). So, one of the protective way against harmful effect of ethanol on gastric mucosal is try to keep the normal level of nitric oxide to stop infiltration of the neutrophil.

The present study showed that the NO reduction induced by ethanol was significantly attenuated by FM that increase gastric NO level in both doses $(\mathrm{P}<0.001)$. This result is consistent with the present histological results that have revealed protection of the gastric mucosa and inhibition of leukocyte infiltration in rats pre-treated with FM. Similar results were reported by Kobayashi et al., (2001) who stated that the inhibition of neutrophil infiltration in the ulcerated gastric tissue is a protective effect against mucosal lesions. The neutrophils are a main source of inflammatory mediators, therefore, suppression of neutrophil infiltration during inflammation was found to improve gastric ulcer healing.

On the other hand, it has been suggested that gastric ulcers are considered to be the manifestation of an inflammatory response (Kang et al., 2014). It is characterized by the migration of polymorphonuclear leukocytes and macrophages in the ulcer area. Migrated macrophages release proinflammatorycytokines such as TNF- $\alpha$ and this upregulates leukocyte recruitment and transmigration in gastric inflamed areas. Our experimental results are in line with these previous data. Ethanol ingestion upregulated the inflammatory response as evidenced by the increase of gastric proinflammatory TNF- $\alpha(\mathrm{P}<0.001)$. These findings are consistent with previous reports (Liu et al., 2012; Li et al., 2013). TNF- $\alpha$ has been closely linked to gastric inflammation via activation of immune cells, generation of other proinflammatory mediators and overexpression of NF- $\kappa \mathrm{B}$ (Li et al., 2013). TNF- $\alpha$ also subdues gastric microcirculation around ulcerated mucosa and thus delays its healing (Hasgulet al., 2014).

Interestingly, FM significantly suppressed gastric TNF- $\alpha$ in both doses $(\mathrm{P}<0.001)$. Thus, the reduction of TNF- $\alpha$ which was accompanied with an elevation of gastric NO, has been viewed as a successful strategy for the management of gastric injury. Meanwhile, the above findings reinforced the histopathological results that described attenuation of neutrophil infiltration, edema and hemorrhage by new formulated milk tablet in ethanol-induced ulcer in rat.

The liver is the main metabolic organ for ingested alcohol; therefore, the series of events that occur in the liver following alcohol intake may predispose the organ to damage. Thus, biochemical markers in the liver and plasma have been widely used as effective methods for the early diagnosis of liver diseases and to determine the functional components for prevention of hepatic lesions. In contrast to the previous studies (Gramenziet al., 2006; Purohitet al., 2009) that reported an elevatedplasma activityof AST and ALT in alcohol-treated rats, our results demonstrate that AST, ALT, and ALP activity was insignificantly induced by ethanol ingestion. This result could be attributed to that observation of plasma AST and ALT induced by ethanol have been dependent on the animal strain, feeding period and method, and/or dosage of ethanol (Baldiet al., 1993). Nevertheless, our data show that pretreatment with high dose of FM lowered the activity of serum AST $(\mathrm{P}<0.05)$. Notably, the present data showed that pretreatment with low dose of FM significantly $(\mathrm{P}<0.05)$ increase in uric acid and creatinine levels. This could be an indicative of the renal cellular injury due to kidney dysfunction and with alteration in membrane permeability.

\section{Conclusion:-}

In conclusion, Based on the above mentioned data, oxidative stress has been involved in the development of ethanolinduced gastric injury where a store of reactive oxygen species (ROS) generated by leukocyte activation triggers mucosal damage via lipid peroxidation and via the antioxidant defenses depletion. Activation of neutrophils is associated with an unregulated inflammatory response with an increased gastric generation of proinflammatory cytokines, including tumour necrosis factor- $\alpha$ (TNF- $\alpha$ ). The current study highlights evidences for the anti-ulcer effect of new formulated milktablet (FM) could be attributed to the its marked antioxidant features abolish lipid peroxidation and preserve gastric antioxidant defenses .It also acts by boosting of NO biosynthesis accompanied reduction of neutrophil infiltration with the suppression of the proinflammatory cytokine TNF- $\alpha$. 
Table 1:- Chemical composition of the new formulated milk tablet (FM) ingredients.

\begin{tabular}{|c|c|c|c|c|c|}
\hline Chemical composition & Fat & Protein & Carbohydrate & Ash & Moisture \\
\hline Skim milk powder & 0.90 & 36.0 & 48.0 & 8.5 & 03.0 \\
Honey & 01.5 & 12.0 & 16.0 & 0.6 & 58.0 \\
Bee pollen & 06.0 & 20.0 & 40.0 & 2.2 & 07.5 \\
Palm pollen & 20.5 & 31.5 & 14.0 & 4.5 & 28,8 \\
Royal jelly & 03.0 & 17.0 & 18.0 & 2.0 & 57.0 \\
Propolis & 15.5 & 25.0 & 28.0 & 4.5 & 10.0 \\
Ginseng & 1.20 & 12.0 & 75.0 & 7.0 & 11.0 \\
\hline
\end{tabular}

Table 2:- Formulations of the new formulated milk tablet(FM) from mixture design.

\begin{tabular}{|c|c|c|c|}
\hline Formulation & Skim Milk powder ( \%) & Honey ( \%) & Other ingredients*( \%) \\
\hline A & 50 & 28 & 22 \\
B & 55 & 23 & 22 \\
C & 60 & 18 & 22 \\
D & 65 & 13 & 22 \\
E & 70 & 8 & \\
\hline
\end{tabular}

* Other ingredients: bee pollen, palm pollen, Royal jelly, propolise and gensing

Table 3:- Sensory evaluation of the new formulated milk tablet (FM) formulation.

\begin{tabular}{|c|c|c|c|c|c|}
\hline Formulation & $\begin{array}{c}\text { Body \&Texture } \\
(\mathbf{5 0})\end{array}$ & $\begin{array}{c}\text { Flavor } \\
\mathbf{( 3 0 )}\end{array}$ & $\begin{array}{c}\text { Color } \\
(\mathbf{1 0})\end{array}$ & $\begin{array}{c}\text { Sweetness } \\
(\mathbf{1 0})\end{array}$ & $\begin{array}{c}\text { Total } \\
\mathbf{( 1 0 0 )}\end{array}$ \\
\hline A & 40 & 22 & 7 & 7 & 76 \\
B & 42 & 25 & 8 & 9 & 83 \\
C & 48 & 28 & 9 & 8 & 94 \\
D & 45 & 25 & 8 & 5 & 86 \\
E & 40 & 18 & 7 & & 70 \\
\hline
\end{tabular}

Table 4:- Chemical composition of the selected formula (C) of new formulated milk tablet (FM)

\begin{tabular}{|l|c|}
\hline Constituent (\%) & FM Formula \\
\hline Fat & 4.20 \\
Protein & 33.85 \\
Carbohydrate & 40.75 \\
Ash & 12.55 \\
Moisture & 7.65 \\
\hline
\end{tabular}


(a)

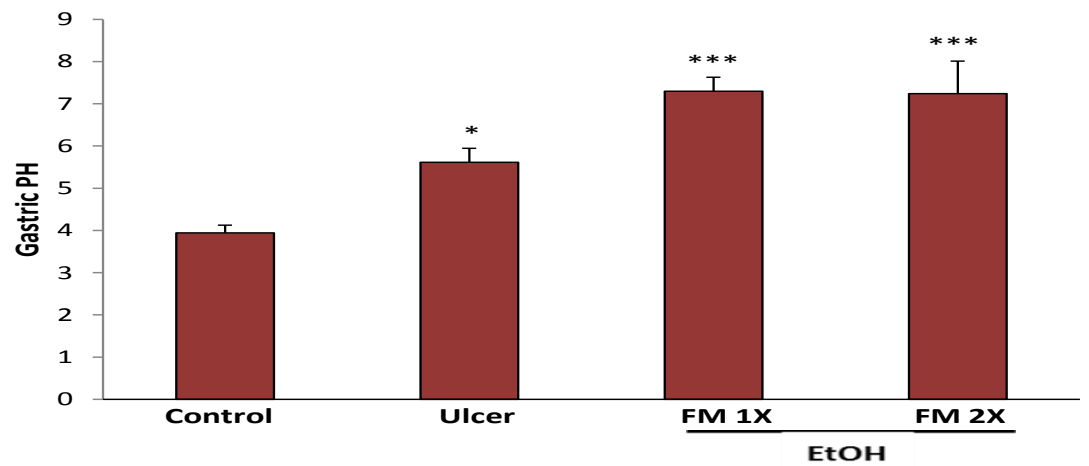

(b)

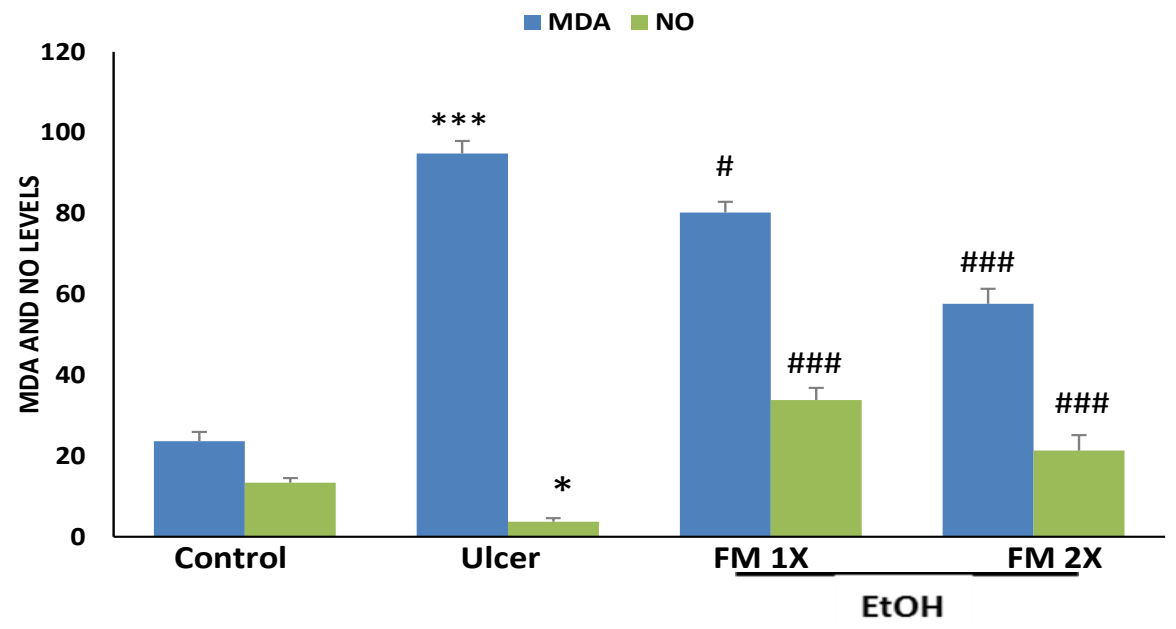

(c)

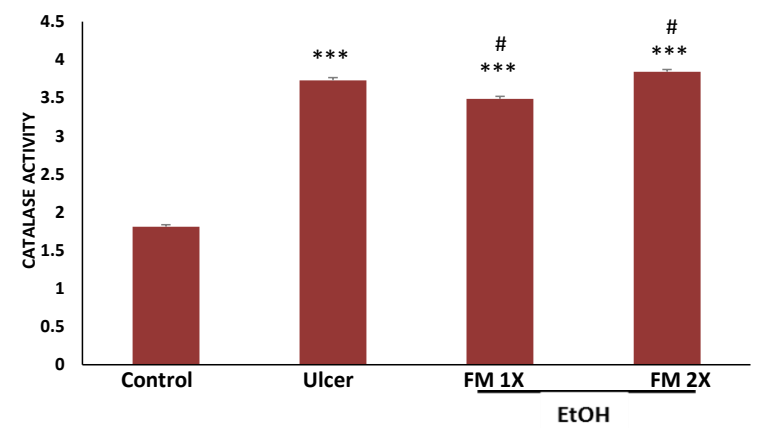

(d)

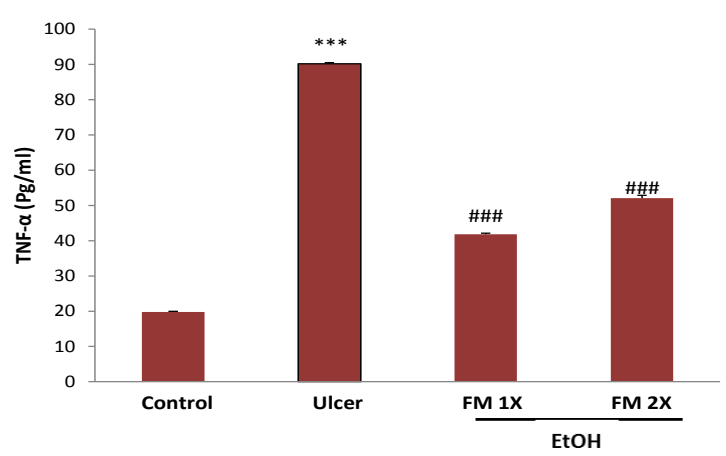

Fig.1:- Effects of FM on gastric pH (a), MDA (nmol/g tissue) and NO ( $\mu \mathrm{mol} / \mathrm{g}$ tissue) (b), CAT (U/L) (c) and Plasma level of TNF- $\alpha(\mathrm{d})$ in EtOH induced gastric ulcer. The data represent the means \pm SEM. $* P<0.05$, $* * * P<$ 0.001 . \# Significantly different from the respective ulcer group at ${ }^{\#} \mathrm{P}<0.05,{ }^{\# \#} P<0.001 . \mathrm{FM}-1 \mathrm{X}(157 \mathrm{mg} / \mathrm{kg}), \mathrm{FM}-$ $2 \mathrm{X}(314 \mathrm{mg} / \mathrm{kg})$. 
(a)

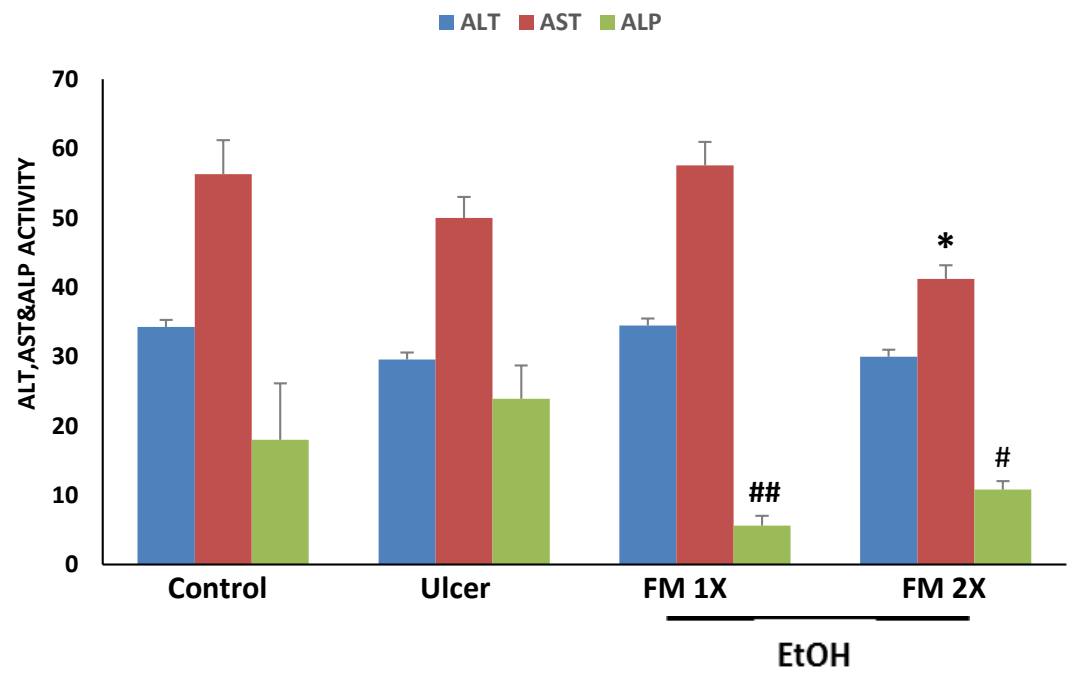

(b)

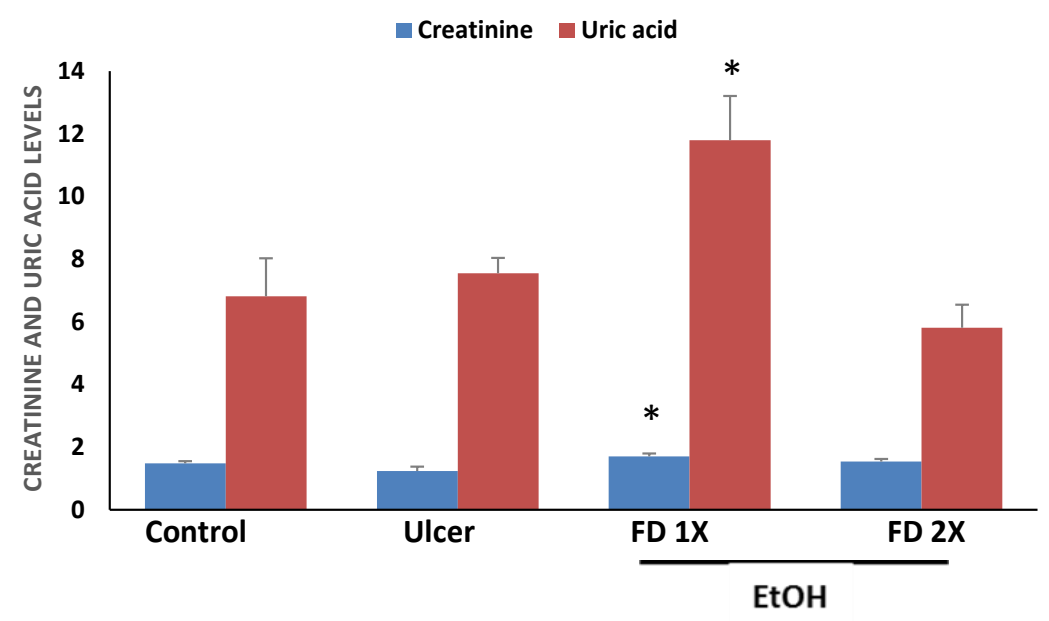

Fig.2:- Effects of FM on the activity of ALT, AST, and ALP (U/L) (a). Creatinine, and uric acid levels (b) in $\mathrm{EtOH}$ induced gastric ulcer. The data represent the mean \pm standard error. $* P<0.05$.\# Significantly different from the respective ulcer group at ${ }^{\#} \mathrm{P}<0.05,{ }^{\# \#} P<0.01 . \mathrm{FM}-1 \mathrm{X}(157 \mathrm{mg} / \mathrm{kg}), \mathrm{FM}-2 \mathrm{X}(314 \mathrm{mg} / \mathrm{kg})$ 

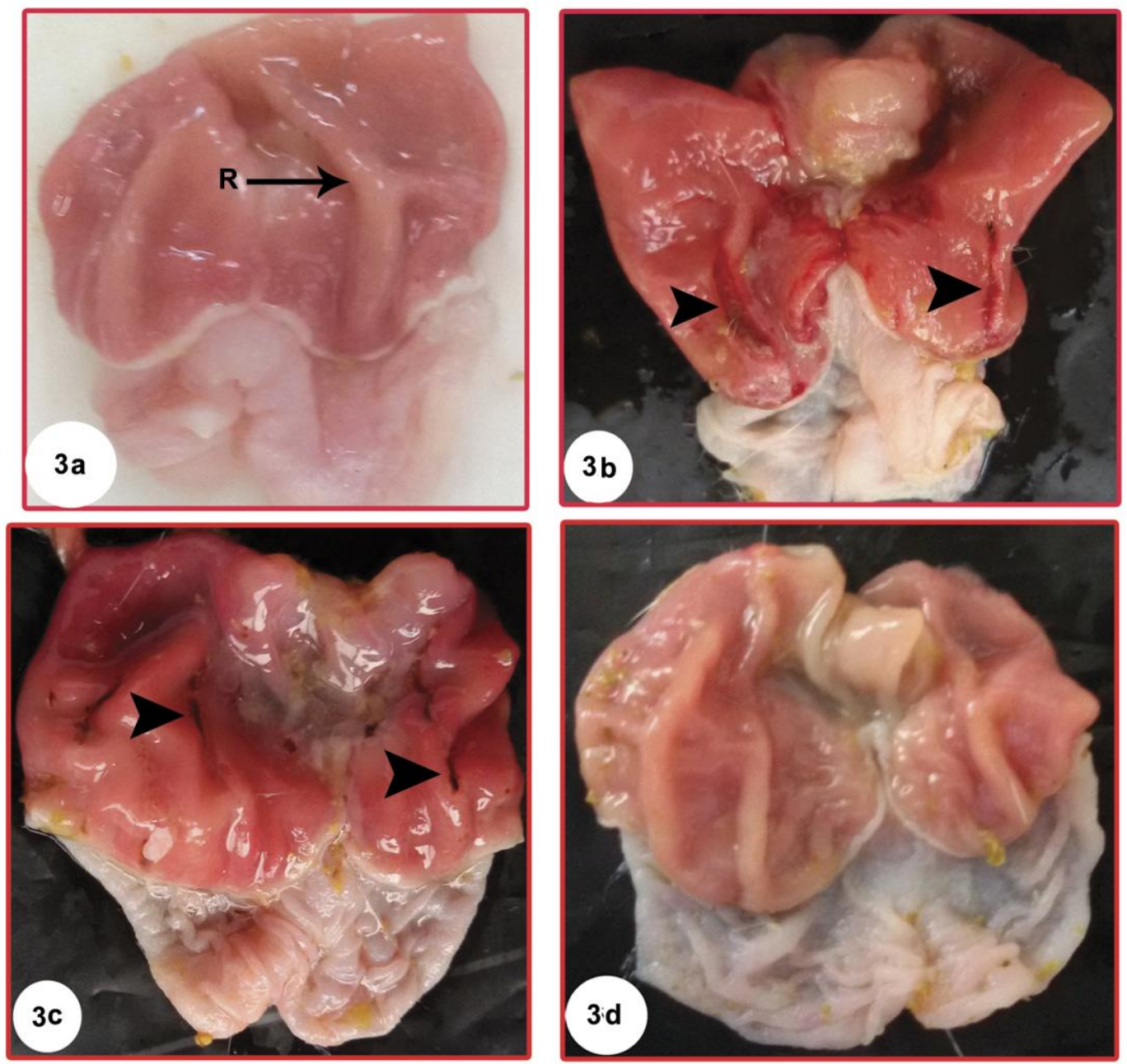

Fig. 3:-Photographs of the stomach of rats (a) the control group showing normal mucosa with rugae, (b) the ulcer group showing linear hemorrhagic bands (arrow heads), (c) the FD pre-treated group (standard diet) showing few hemorrhagic areas (arrow heads) and (d) the FD pre-treated group (double standard diet) showing normal stomach architecture. 

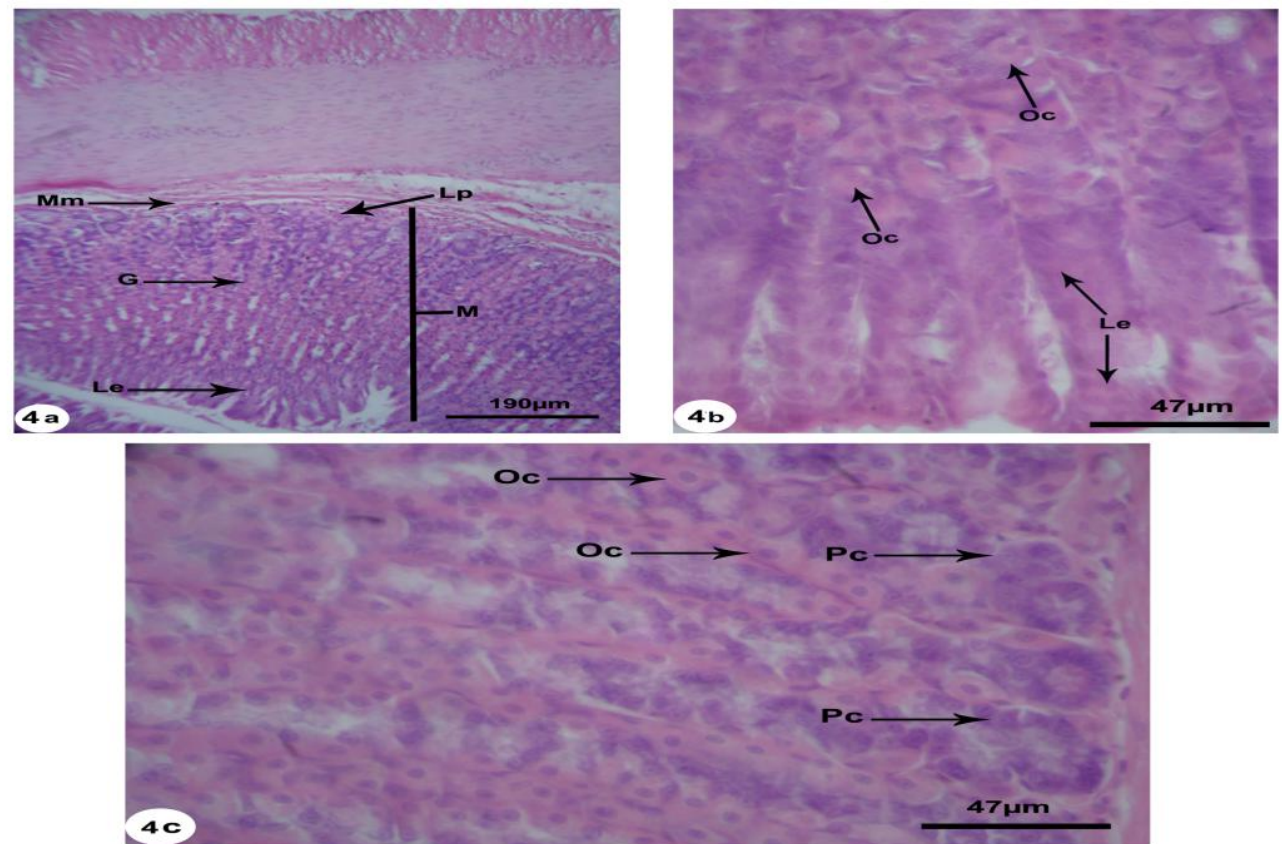

Fig. 4 (a-c):-photomicrographs of the stomach of rats of the control group showing normal mucosal architecture. The gastric mucosa (M), lining epithelium (Le), lamina propria (Lp), muscularis mucosae (Mm), gastric gland $(\mathrm{G})$, oxyntic cells $(\mathrm{Oc})$ and peptic cells $(\mathrm{Pc})$.
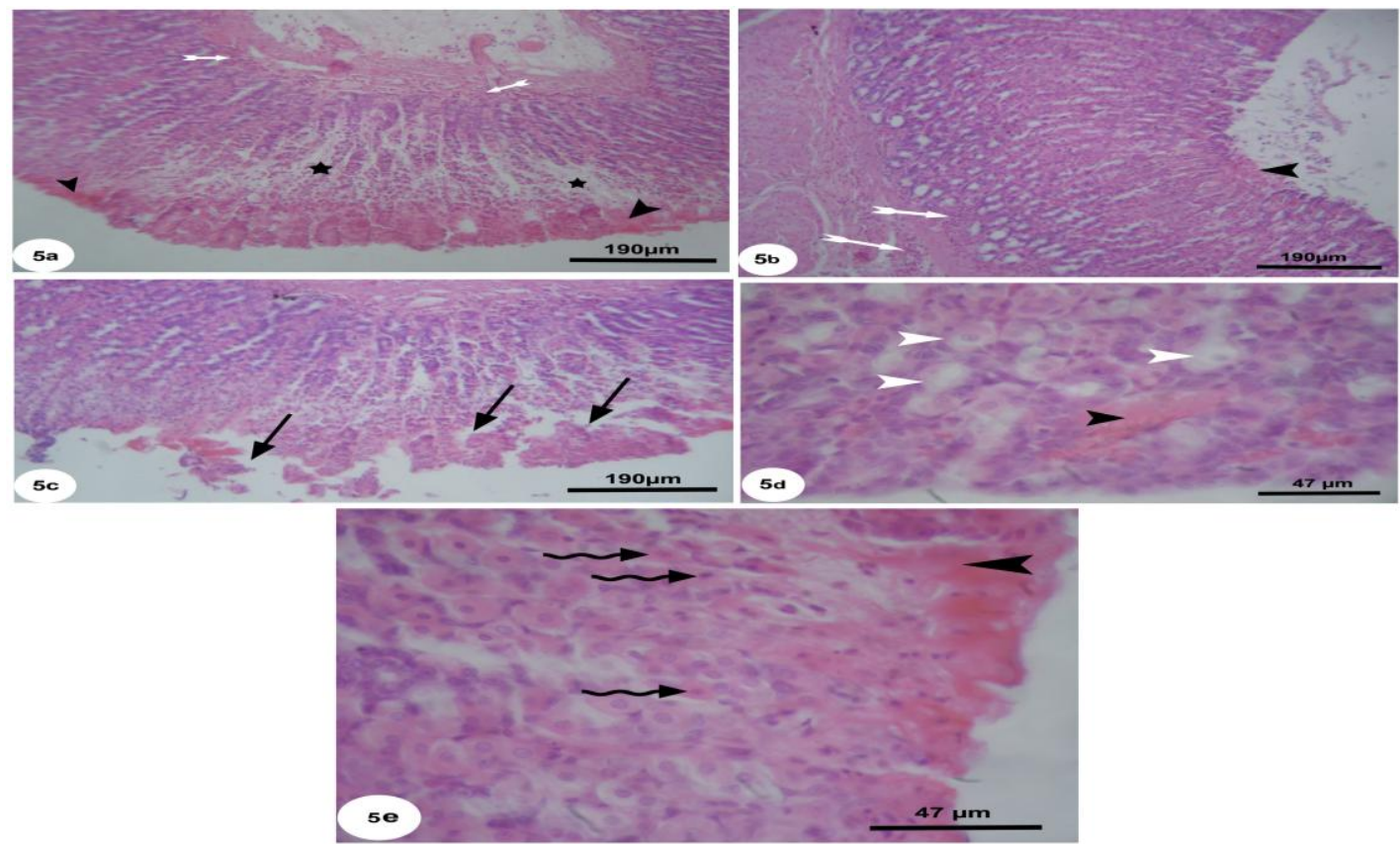

Fig. 5 (a-e):-photomicrographs of the stomach of rats of the ulcer group showing the gastric mucosal lesions. The necrotic mucosa (asterisk), mucosal hemorrhage (black arrow head), leucocytes infiltration (white arrow), epithelial lifting (black arrow), cell necrosis (white arrow head) and pyknotic nuclei (curved arrow). 

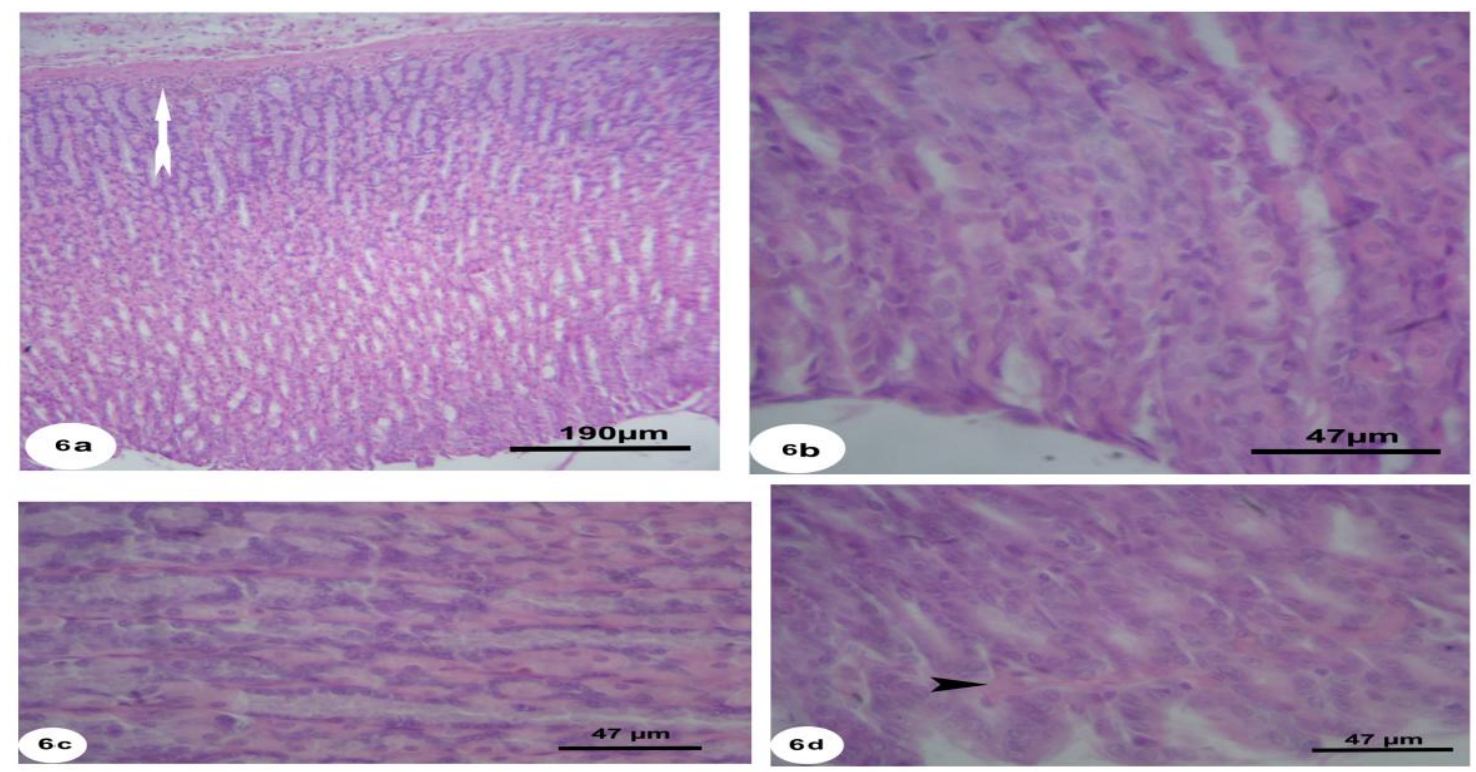

Fig. 6 (a-d) :-photomicrographs of the stomach of rats of the FD pre-treated group (standard diet) showing normal mucosal architecture with superficial gastric lesions. The leucocytes infiltration (white arrow) and mucosal hemorrhage (black arrow head).

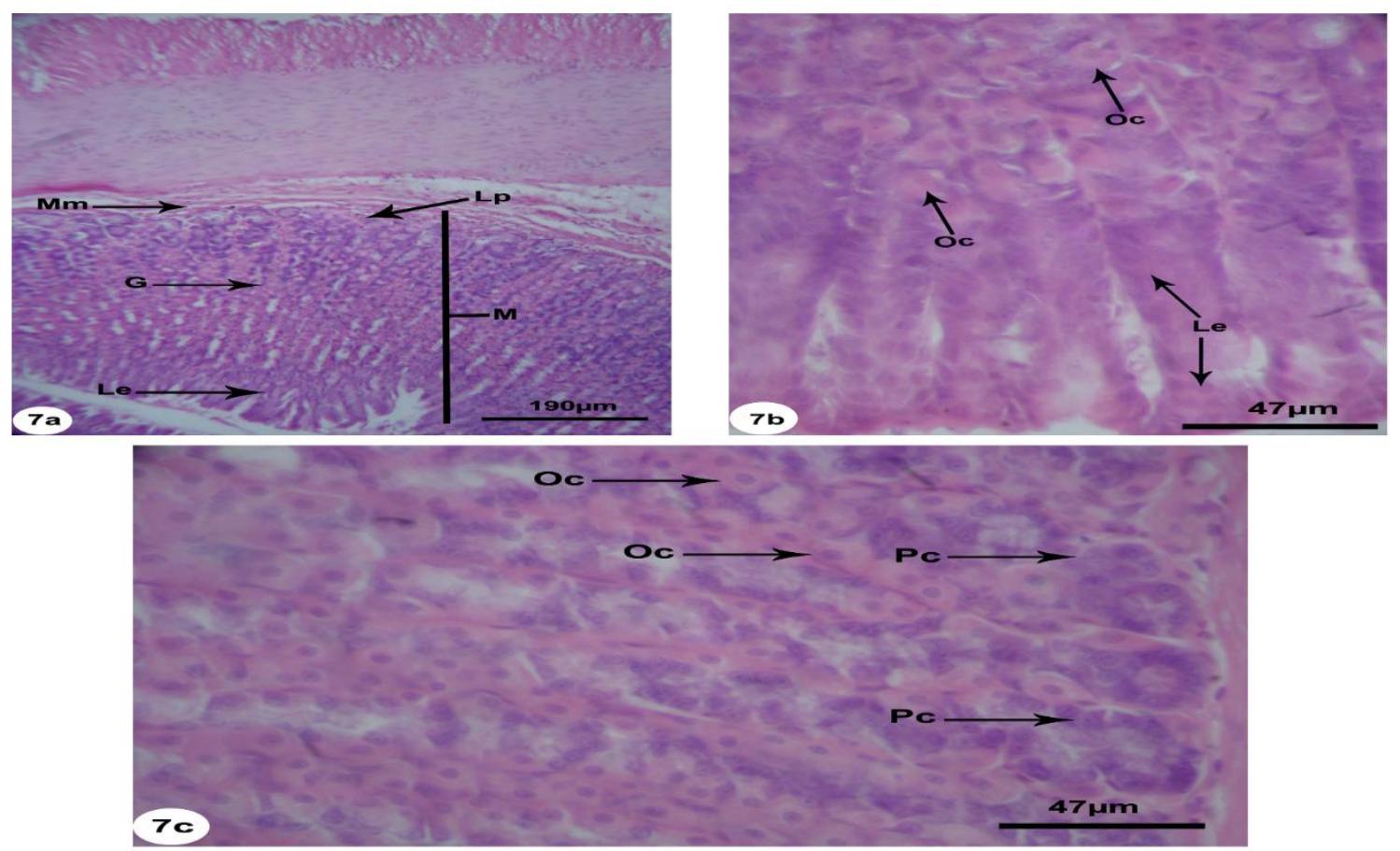

Fig. 7 (a-c) :-photomicrographs of the stomach of rats of the FD pre-treated group(double standard diet) showing normal mucosal architecture. 


\section{Reference:-}

1. Brown DM, Everhart JE. Cost of digestive diseases in the United States. In Digestive diseases in the United States: epidemiology and impact. Everhart JE. Washington, DC. National Institutes of Health Publication 1994; 57-82.

2. Sandler RS, Everhart JE, Donowitz M, Adams E, Cronin K, Goodman C, Gemmen E, Shah S, Avdic A, Rubin R. The burden of selected digestive diseases in the United States. Gastroenterol 2002; 122: 1500-1511.

3. Calam J, Baron JH. Pathophysiology of duodenal and gastric ulcer and gastric cancer. Brit Med J 2001; 323:980-983.

4. Nemeroff CB, Hernandez DE, Orlando RC, Prange AJ. Cytoprotective effect of centrally administered neurotensin on stress-induced gastric ulcers. Am J Physiol 1982; 242:G342-346.

5. Franke A, Teyssen S, Singer MV. Alcohol-related diseases of the esophagus and stomach. Dig Dis 2005 ; 23 (34):204-213.

6. Al Batran R, Al-Bayaty F, Jamil AMM, Abdualkader AM, Hadi HA, Ali HM, Abdulla MA. In vivo antioxidant and antiulcer activity of Parkiaspeciosaethanolic leaf extract against ethanol-induced gastric ulcer in rats. PLoS One 2013;8 (5):e64751.

7. Mei X, Xu D, Xu S, Zheng Y. Novel role of $\mathrm{Zn}(\mathrm{II})$-curcumin in enhancing cell proliferation and adjusting proinflammatory cytokine-mediated oxidative damage of ethanol-induced acute gastric ulcers. ChemBiol Interact. 2012; 197(1):31-39.

8. Sangiovanni E, Vrhovsek U, Rossoni G, Colombo E, Brunelli C, Brembati L, Trivulzio S, Gasperotti M, Mattivi F, Bosisio E, Dell'Agli M. Ellagitannins from Rubus berries for the control of gastric inflammation: in vitro and in vivo studies. PLoS One 2013; 8(8), e71762.

9. Loguercio C, Taranto D, Beneduce F, Vecchio BC, Vincentilis A, Nardi G, RomanoM. Glutathione prevents ethanolinduced gastric mucosal damage and depletion of sulfhydryl compounds in humans. Gut 1993; 34:161-5.

10. Liu Y, Tian X, Gou L, Fu X, Li S, Lan N, Yin X.Protective effect of 1-citrulline against ethanol-induced gastric ulcer in rats. Environ ToxicolPharmacol 2012;34(2): 280-287.

11. Salga MS, Ali HM, Abdulla MA, Abdelwahab SI. Gastroprotective activity and mechanism of novel dichloridozinc(II)-4-(2-(5-methoxybenzylideneamino)ethyl) piperazin-1-iumphenolat e complex on ethanol- induced gastric ulceration. ChemBiol Interact 2012; 195(2):144-153.

12. Halabi MF, Shakir RM, Bardi DA, Al-Wajeeh NS, Ablat A, Hassandarvish P, Hajrezaie M, Norazit A, Abdulla MA. Gastroprotective activity of ethyl-4-((3,5-di-tert-butyl-2-hydroxy benzy- lidene) amino)benzoate against ethanolinduced gastric mucosal ulcer in rats. PLoS One 2014; 9(5):e95908.

13. Antonisamy P, Subash-Babu P, Alshatwi AA, Aravinthan A, Ignacimuthu S, Choi KC, etal. Gastroprotective effect of nymphayol isolated from Nymphaeastellata (Willd.) flowers: Contribution of antioxidant, anti-inflammatory and antiapoptotic activities. ChemBiol Interact 2014; 224: 157-163.

14. Livney, Y.D., 2010. Milk proteins as vehicles for bioactives, Current Opinion in Colloid \& Interface. Science. 15, 7383.

15. Zaghloul A, El-Shattawy H, Kassem A. Honey, a prospective antibiotic: extraction, formulation, and stability.Pharmazie 2001; 56:643-647.

16. Khalil MI, Sulaiman SA and Boukraa L. Antioxidant Properties of Honey and Its Role in Preventing Health Disorder. The Open Nutraceuticals Journal 2010; 3: 6-16.

17. Martín-Muñoz M F, Bartolome B, Camiona M, Bobolea I, Garcia A M C, Quirce S. Bee pollen: a dangerous food for allergic children. Identification of responsible allergens. AllergologiaImmunopathologia 2010; 38: $263-265$.

18. Fernández M I. Constituents of a hexane extract of Phoenix dactylifera. Photochemistry 1983; 22 (9): $207-2088$.

19. Moritz RFA, Southwick E E. Bees a super organisms. An evolutionary reality, first ed., Springer-Verlag, Berlin; 1992.

20. Mărghitaş L A. Produseleapicoleşiprincipalele Med. 2008;82: 384-385.

21. Özcan M, Ayar A. Effect of propolis extracts on butter stability. J Food Quality 2003; 26 (1): 65-73.

22. Kamal S S, Kaur D, Singh S, Sharma A, Katual M K, Garg A K, Kumar R. An Investigative and Explanatory Review on Use of Milk as a Broad-Spectrum Drug Carrier for Improvement of Bioavailability and Patient Compliance. J Young Pharmacists. 2016; 8(2):72-75.

23. Sun Y. Structure and biological activities of the polysaccharides from the leaves, roots and fruits of Panax ginseng CA Meyer: An overview. CarbohydrPolym 2007; 85: 490-499.

24. Uno K, Nicholls SJ. Biomarkers of inflammation and oxidative stress in atherosclerosis. Biomark Med 2010; 4: 361373.

25. AOAC.,2006. Official Methods of Analysis, 18thed. Association of the Official Analytical Chemists.Washington, D.C., USA.

26. Huang C-C, Chen Y-M, Wang D-C, Chiu C-C,Lin W-T, Huang C-Y, Hsu M-C. Cytoprotective Effect of American Ginseng in a Rat EthanolGastric Ulcer Model. Molecules. 2014; 19: P 316-326.

27. Sabiu S, Garuba T, Sunmonuc T, Ajania E, Sulymana A, Nuraina I, Balogun A. Indomethacin-induced gastric ulceration in rats: Protectiveroles of Spondiasmombin and Ficus exasperate. Toxicology Reports 2015; 2: $261-267$.

28. Humason G L. Animal Tissue Techniques. 3rd edition. W. H. Freeman Company, San Francisco; 1972. 
29. Arab H H, Salama S A, Omar H A, Arafa E A, Maghrabi I A. Diosmin Protects against Ethanol-Induced Gastric Injury in Rats: Novel Anti-Ulcer Actions. PLoS ONE 2015; 10(3):e0122417.

30. Schreiber S, ScheidP. Gastric mucus of the guinea pig: proton carrier and diffusion barrier, The Am J PhysiolGastrointest Liver Physiol 1997; 272(1): G63-G70.

31. Lüllmann H, Mohr K, Ziegler A, Bieger D. Color atlas of pharmacology, 2nd ed., Thieme Stuttgart, New York, pp. 166. 2000.

32. Schlorff EC, Husain K, Somani SM. Dose- and time-dependent effects of ethanol on plasma antioxidant system in rat. Alcohol 1999;17:97-105

33. Recknagel RO, Ghoshal AK. Quantitative estimation of peroxidative degeneration of rat liver microsomal and mitochondrial lipids after carbon tetrachloride poisoning. ExpMolPathol 1996; 5:413-26.

34. Zakaria Z A, Balan T, Mamat SS, Mohtarrudin N, Kek T L, Salleh M Z. Mechanisms of gastroprotection of methanol extract of Melastomamalabathricum leaves. BMC Complement. Altern Med 2015; 15: 135.

35. J'unior F E B, De Oliveira D R, Boligon A A, Athayde ML, Kamdem JP, Macedo GE, da Silva GF, de Menezes IR, Costa JG, Coutinho HD, Kerntopf MR, Posser T. Protective effects of Croton campestris A. St-Hill in different ulcer models in rodents: evidence for the involvement of nitric oxide and prostaglandins. J Ethnopharmacol 2014; 153(2): 469-477.

36. Nordin N, Salama S M, Golbabapour S, Hajrezaie M, Hassandarvish P, Kamalidehghan B, Majid NA, Hashim NM, Omar H, Fadaienasab M, Karimian H, Taha H, Ali HM, Abdulla MA. Antiulcerogenic effect of methanolic extracts from Enicosanthellumpulchrum (King) Heusden against ethanol-induced acute gastriclesion in animal models. PLoS ONE 2014; 9(11): e111925

37. O’Donnell VB, ChumleyPH, Hogg N, Bloodsworth A, Darley-Usmar VM, Freeman BA. Nitric oxide inhibition of lipid peroxidation: Kinetics of reaction with lipid peroxyl radicals and comparison with alpha-tocopherol. Biochemistry 1997; 36: 15216-23.

38. Calatayud S, Barrachina D, Esplugues JV. Nitric oxide: relation to integrity, injury, and healing of the gastric mucosa. Microsc Res Tech 2001; 53:325-35.

39. Wallace J L, McKnight W, Wilson TL, del Soldato PD, CirinoG.Reduction of shock-induced gastric damage by a nitric oxide-releasing aspirin derivative: role of neutrophils. Am J Physiol 1997; 273(6): G1246-G1251.

40. Abdulla MA, Ali HM, Ahmed A, Noor SM, Ismai 1. Evaluation of the anti-ulcer activities of Morusalba extracts in experimentally-induced gastric ulcer in rats. Biomed Res India 2009; 20: 35-39.

41. Goswami M, Kulshreshtha M, Rao CV, Yadav S. Anti-ulcer potential of Lawsoniainermis L. Leaves against gastric ulcers in rats. J appl pharmacy sci 2011; 1: 69-72.

42. Kobayashi T, Ohta Y, Yoshino J, Nakazawa S. Teprenone promotes the healing of acetic acid-induced chronic gastric ulcers in rats by inhibiting neutrophil infiltration and lipid peroxidation in ulcerated gastric tissues. Pharmacol Res 2001; 43: 23-30.

43. Kang J-W, Yun N, Han H-J, Kim J-Y, Kim J-Y, S-M Lee1.Protective Effect of FlosLonicerae against Experimental Gastric Ulcers in Rats: Mechanisms of Antioxidant and Anti-Inflammatory Action. Evid Based Complement Alternat Med 2014; 24: 2014-596920.

44. Li W, Huang H, Niu X, Fan T, Mu Q, Li H. Protective effect of tetrahydrocoptisine against ethanol induced gastric ulcer in mice. ToxicolApplPharmacol 2013; 272(1): 21-29.

45. Hasgul R, Uysal S, Haltas H, Akyol S, Yuksel Y, Gurel A, Armutcu F. Protective effects of Ankaferd blood stopper on aspirin-induced oxidative mucosal damage in a rat model of gastric injury. ToxicolIndHealth , 2014;30(10):888895.

46. Gramenzi A, Caputo F, Biselli M, Kuria F, Loggi E, Andreone P, Bernardi M. Alcoholic liver disease: pathophysiological aspects and risk factors. Aliment PharmacolTher 2006; 24:1151-1161.

47. Purohit V, Gao B, Song BJ. Molecular mechanisms of alcoholic fatty liver. Alcohol ClinExp Res 2009; 33:191-205.

48. Baldi E, Burra P, Plebani M, Salvagnini M. Serum malondialdehyde and mitochondrial aspartate aminotransferase activity as markers of chronic alcohol intake and alcoholic liver disease. Ital J Gastroenterol 1993; 25:429-432. 